\title{
PAX-8 expression in renal tumours and distant sites: A useful marker of primary and metastatic renal cell carcinoma?
}

\author{
Meaghan L Barr, ${ }^{1}$ Lucia B Jilaveanu, ${ }^{1}$ Robert L Camp, ${ }^{2}$ Adebowale J Adeniran, ${ }^{2}$ \\ Harriet M Kluger, ${ }^{1}$ Brian Shuch $^{3}$
}

- Additional material is published online only. To view please visit the journal online (http://dx.doi.org/10.1136/ jclinpath-2014-202259).

${ }^{1}$ Division of Oncology, Department of Medicine, Yale School of Medicine, New Haven, Connecticut, USA ${ }^{2}$ Department of Pathology, Yale School of Medicine, New Haven, Connecticut, USA

${ }^{3}$ Department of Urology, Yale School of Medicine, New Haven, Connecticut, USA

\section{Correspondence to} Dr Brian Shuch, Department of Urology, Yale School of Medicine, 310 Cedar Street, 238A, New Haven, CT 06510, USA; Brian.shuch@yale.edu

Received 26 February 2014 Revised 1 September 2014 Accepted 22 September 2014 Published Online First 14 October 2014

CrossMark

To cite: Barr $\mathrm{ML}$, Jilaveanu LB, Camp RL, et al. I Clin Pathol 2015;68:12-17.

\section{ABSTRACT}

Aims Immunohistochemical stains have greatly improved the diagnostic accuracy of renal cell carcinoma (RCC) for primary and distant tumours. We evaluate a marker that has recently been incorporated in clinical practice, PAX-8, in primary and metastatic RCCs. Methods Two distinct tissue microarrays were used, one consisting of over 334 renal tumours, 294 with adjacent normal kidney and the other with 40 matched nephrectomy and metastatic sites of RCC. PAX-8 expression was assessed by a method of quantitative immunofluorescence.

Results PAX-8 was positive in 96\% (146/152) of normal renal tissue and 83\% (227/272) of renal tumours. PAX-8 staining was positive in clear cell, papillary and chromophobe tumours in 80\% (165/207), $95 \%(39 / 41)$ and $100 \%(6 / 6)$ of samples, respectively. Overall, intensity of PAX-8 expression was significantly higher in RCC metastatic sites than in the primary site $(p=0.0047)$, however, in matched sites there was no statistically significant difference in the proportion of positive versus negative specimens $(p=0.274)$.

Conclusions As the role of molecular markers expands in the diagnostic algorithm, this study confirms that PAX-8 expression is a useful diagnostic marker for RCC. PAX-8 expression was found in the primary tumour and distant sites. Compared with normal tissue and other histological types, clear cell RCC has lower PAX-8 expression and is less frequently positive, therefore, the lack of expression does not exclude a tumour of renal origin.

\section{INTRODUCTION}

Determining a cancer's origin has significant implications as it generally determines the local treatment strategy or choice of systemic therapy. Since there are multiple new agents approved for the treatment of metastatic renal cell carcinoma (RCC), it is of critical importance to correctly determine the tumour type prior to embarking down a treatment pathway. Unfortunately relying on a diagnostic core biopsy is often challenging due to limited availability of material from often difficult to sample metastatic sites and therefore Current National Comprehensive Cancer Guidelines do not make strong recommendations on the role of biopsy. ${ }^{1}$ Even when resected, as high as 5\% of primary tumours are considered unclassified since they may be too poorly differentiated, have sarcomatoid differentiation without evidence of epithelial components, or have a mixed morphological pattern. For metastatic tumours that arise from the kidney, these lesions may resemble multiple other malignancies (ex. other clear cell neoplasms), have different characteristics than the prior primary tumour or be poorly differentiated. ${ }^{2}$ Moreover, these tumours are often necrotic, resulting in additional diagnostic challenges. For these reasons pathologists often rely on immunohistochemistry to distinguish primary and metastatic RCCs from other primary tumours. ${ }^{3}$ However, it has increasingly been recognised that RCC is not one disease, but a heterogenous group of cancers with a distinct genetic basis. Nearly all RCC subtypes can metastasise and therefore, in the setting of a carcinoma of unknown origin, it is extremely useful to have a reliable diagnostic marker with excellent specificity and sensitivity. ${ }^{4}$

Several immunohistochemistry panels have proven useful in identifying primary or metastatic RCC. ${ }^{4-7}$ Commonly used markers include cytokeratins, vimentin, RCC marker, CD10 and carbonic anhydrase 9 (CAIX). ${ }^{6}$ While CAIX is an excellent marker for clear cell RCC, which results from the loss of the VHL protein, it may not be an ideal marker in non-clear cell subtypes. Moreover, it can be physiologically expressed as a result of hypoxia, further diminishing its specificity. ${ }^{8}$

PAX-8 and PAX-2 have been investigated as markers for RCC. Both belong to a group of paired box gene family of transcription factors involved in organogenesis. PAX-8 regulates renal and thyroid organogenesis. ${ }^{9-12}$ During embryogenesis, PAX-8 is coexpressed with another member of its gene family, PAX-2. The expressions of these proteins are separated temporally during development. Together, these proteins may determine nephric lineage. ${ }^{9}$ In the adult kidney, PAX- 8 has been detected in the renal epithelial cells of the renal tubules and in Bowman's capsule. ${ }^{13}$ Recent studies suggest that PAX-8 is a specific marker for primary and metastatic RCC (mRCC). ${ }^{13-22}$ When distinguishing between a primary and metastatic kidney tumour, PAX-8 could very useful. In an analysis of over 1100 various tumour types, the specificity and sensitivity of this marker was excellent to distinguish RCC and ovarian cancer from other malignancies. ${ }^{22}$ PAX-8 is also useful for distinguishing RCC from the second most common type of kidney tumour, urothelial carcinoma, as $>90 \%$ of cases have no expression of this marker. ${ }^{14}$ In an analysis of multiple renal malignancies arising in the kidney including medullary and collecting duct RCC, PAX-8 was able to reliably distinguish RCC from urothelial carcinoma unlike other investigated markers including vimentin, high molecular weight cytokeratin, CK7, CD10, CAIX and PAX-2. ${ }^{23}$ 
Many of the prior studies of PAX-8 expression in primary and mRCC have had limitations including the use of small cohorts, a focus on clear cell RCC only, or use of subjective methods of PAX-8 scoring. ${ }^{13-18} \quad 20-22$ To investigate the utility of PAX-8 expression for diagnosing RCC and mRCC, we evaluated its expression in RCC cell lines and in two patient cohorts, one containing multiple RCC subtypes and adjacent normal kidney and the other containing matched primary and metastatic tumours. The use of quantitative immunofluorescence allows an objective measure of PAX-8 expression.

\section{MATERIALS AND METHODS \\ Western blot analysis}

PAX-8 expression was assessed in human RCC cell lines Caki-1, A498, ACHN, 769-P and 786-0; all purchased from American Type Culture Collection (Manassas, Virginia, USA). Protein lysates were obtained by standard methods and $40 \mu \mathrm{g}$ of protein per sample was subjected to gel electrophoresis and membrane transfer. Western blotting, was performed following standard protocols as previously described. ${ }^{24}$ After transfer, the membrane was blocked in 5\% milk and incubated overnight with rabbit polyclonal anti-PAX-8 (Cell Marque, Rocklin, California, USA, 1:500 dilution). To assess protein loading, the membrane was probed with mouse anti- $\beta$-actin (Sigma-Aldrich, St Louis, Missouri, USA, 1:5000 dilution). Protein was visualised using peroxidase-conjugated antirabbit or antimouse $\operatorname{IgG}$ antibody (Jackson ImmunoResearch Laboratories, 1:5000 dilution) and SuperSignal West Pico Chemiluminescent Substrate (ThermoScientific, Waltham, Massachusetts, USA).

\section{Tissue microarray construction}

Two separate tissue microarray (TMA) sets were constructed using archival, surgical material from non-overlapping resected RCC cases that have been described previously. ${ }^{25} 26$ Cohort A consisted of a large series of patients $(n=334)$ with renal tumours resected between 1987 and 1999 and included a total of two $0.6 \mathrm{~mm}$ cores from the tumour. Two hundred and ninety-four of the patients had a matched normal adjacent kidney core. ${ }^{26}$ The cohort included clear cell RCC (71\%), papillary RCC (14\%), oncocytoma (6\%), chromophobe RCC (2\%), as well as unclassified/other histologies (7\%).

'Cohort B' was constructed from 40 separate patients with RCC with metachronous or synchronous metastatic disease treated between 1978 and 2011. The array set included eight $0.6 \mathrm{~mm}$ cores, four from both the primary renal tumour and four from matched metastatic sites, two on each slide. ${ }^{25}$ Clinical data was available for 34 patients. The cohort included clear cell $(91.2 \%)$ RCC and clear cell with sarcomatoid features $(8.8 \%)$ RCC. Sites of metastasis included lung (13), bone (7), soft tissue, skin and lymph node (8), adrenal glands (2), liver (2), colon (1) and pituitary gland (1).

\section{Immunofluorescent staining}

PAX-8 fluorescence was performed using methods that have been previously described. ${ }^{27}$ Overnight incubation with rabbit polyclonal anti-PAX-8 (Cell Marque, Rocklin, California, USA, 1:200 dilution) and goat antirabbit horseradish peroxidaseconjugated secondary antibody (Envision, Dako, Carpinteria, California, USA) was used to detect the target signal. Slides were then incubated for $1 \mathrm{~h}$ in an antibody cocktail of mouse anticytokeratin (Dako, Carpinteria, California, USA, 1:200 dilution), CAIX (gift from Jan Zavada, 1:1000 dilution), CD10 (Dako, Carpinteria, 1:500 dilution) and streptavidin HRP (Sigma-Aldrich, St Louis, Missouri, USA, 1:200 dilution). A goat antimouse horseradish peroxidase-conjugated secondary antibody (Envision, Dako, Carpinteria, California, USA) and Cyanine2-tyramide were used to detect the mask. Slides were incubated in 4,6-diamidine-2-phenylindole (Invitrogen, Carlsbad, California, USA, 1:300 dilution) to identify the nuclei (Invitrogen, Carlsbad, California, USA).

\section{Automated image acquisition and statistical analysis}

TMA studies have been criticised for reproducibility of scoring on a continuous scale, distinguishing between subcellular compartments, and fine discrimination between low levels of expression. A multiparametrical algorithm developed by Camp and colleagues called Automated Quantitative Analysis (AQUA) allows a rapid, automated analysis of the TMA cohort. ${ }^{27}$ One algorithm called pixel-based locale assessment allows the subcompartmental regions to be separately analysed. An additional algorithm allows rapid exponential subtraction to remove any out of focus images that may be in a different plan. These methods have been validated and have a high degree of correlation within blocks of the same TMA as well as when compared with pathologist assessment. ${ }^{24} 26-28$

To obtain a quantitated measurement of protein expression, staining was analysed with an AQUA. ${ }^{27}$ Monochromatic, highresolution $(1024 \times 1024)$ images were captured of each histo spot, as demonstrated in figure 1. Cyanine2-tyramide signal was used to visualise cytoplasmic tissue upon which the tumour mask was based, while the individual nuclei were visualised with 4,6diamidine-2-phenylindole. PAX-8 was visualised with Cyanine5tyramide. AQUA allowed for the evaluation of PAX-8 expression on a continuous scale. Since PAX-8 staining is considered to predominantly stain in the nucleus, ${ }^{20}$ the intensity of the target signal was assayed within the nuclear compartment of the tumour mask to determine each histo spot's AQUA score. The signal intensity was scored from 0 to 255 for each histo spot. In order to establish a cut-off point for PAX-8 positivity, a large representative cohort of AQUA images were provided to a pathologist for review in a blinded fashion. Cases considered PAX-8 positive all had an AQUA score of $>6.0$, and therefore a score of $>6$ was used to define AQUA positivity for PAX-8 expression. Tumour spots were excluded from the analysis if there was abundant necrotic tissue, insufficient tumour cells $(<3 \%$ of the area of the histo spot) or loss of tissue in the spot during immunostaining. ${ }^{28}$

For our data analysis, we evaluated the distribution of PAX-8 expression and found the distribution of the tumour and normal expression to approach normalcy (skewness and kurtosis, 1.78 and 3.15 , tumour, and 1.39 and 2.54 for normal, respectively) (see online supplementary figure S2). To ensure our statistical analyses were valid with parametrical testing, we performed a natural log transformation of the values to improve normalcy (see online supplementary figure S2). Statistical analysis relying on the parametrical testing was performed with the transformed data to confirm that there was no change in the results with our assumption of normalcy (see online supplementary figure S3).

The Pearson correlation test was used to compare AQUA scores for markers from matching spots from the two arrays. The paired $t$ test was used to compare PAX-8 expression between matched tumour and normal samples from patients with distinct RCC subtypes. ANOVA was used to determine if PAX-8 expression differed significantly among all tumour types $(\mathrm{p}<0.0001)$. Post hoc testing using Fisher's protected least significant difference test was used to compare PAX-8 expression in RCC subtypes. ANOVA and $\chi^{2}$ analysis were used to compare PAX- 8 expression between primary and metastatic sites 
Figure 1 Example of Automated Quantitative Analysis (AQUA) staining for PAX-8 in PAX-8-positive (upper panels) and PAX-8 negative (lower panels) histo spots. Slides were incubated in anti-PAX-8 to create target signal. The target signal was visualised using cyanine5-tyramide. To create a tumour mask, slides were incubated in a cytokeratin, carbonic anhydrase 9 (CAIX) and streptavidin HRP cocktail. The tumour mask was visualised with cyanine2-tyramide. Nuclei were visualised using 4,6-diamidine-2-phenylindole (DAPI).

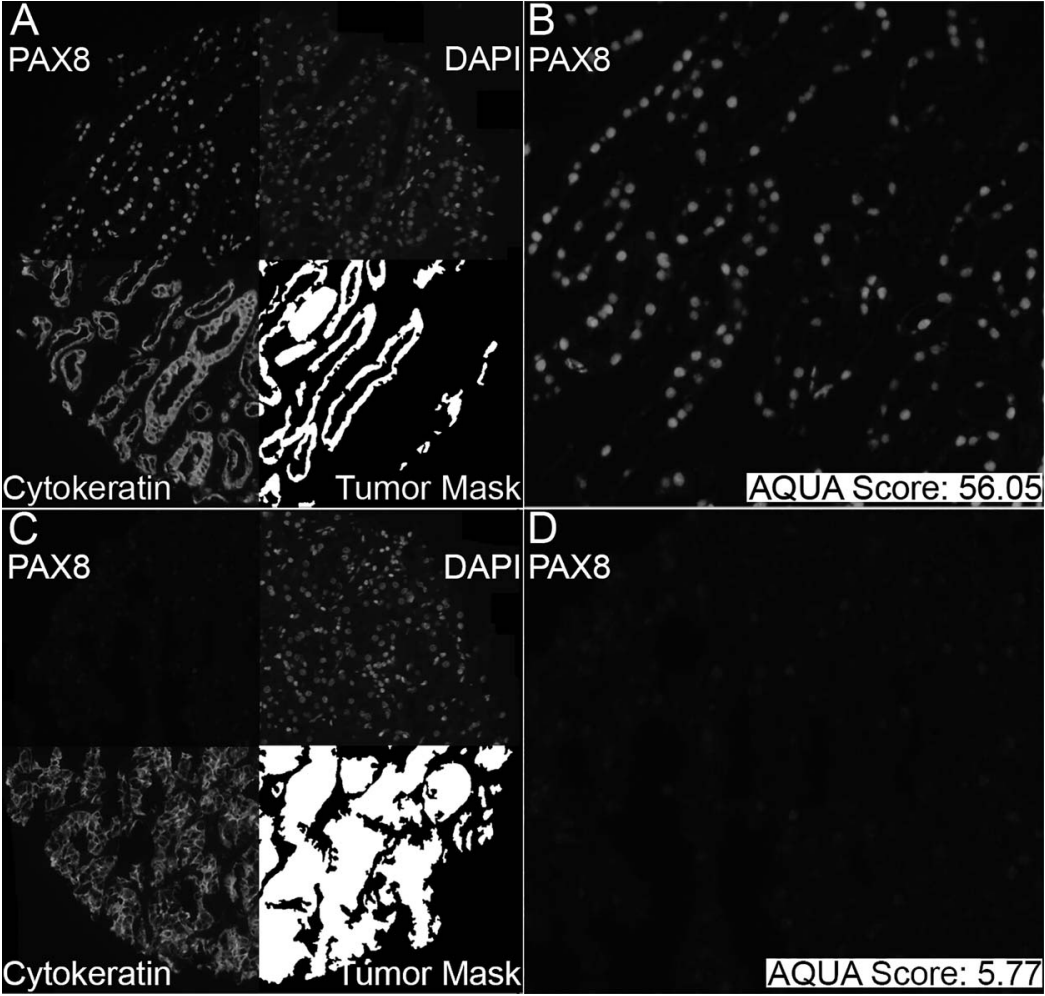

and between primary and matched metastatic samples. Survival time was calculated from date of initial surgery to date of last follow-up, with censoring performed at death from other causes or last alive contact. Survival analyses were performed using the Cox proportional hazards model. Statview and JMP V.9.0 (SAS Institute, Cary, North Carolina, USA) were used to conduct statistical analysis.

\section{RESULTS}

Western blot analysis confirmed PAX- 8 antibody specificity and its presence in various RCC cell lines such as ACHN, A498 and 769-P (see online supplementary figure S1). PAX-8 protein was observed at molecular weights of $48 \mathrm{kD}$ and $58 \mathrm{kD}$, both corresponding with previously described sizes of the various PAX-8 isoforms. ${ }^{29}$ PAX-8 immunofluorescence was assessed in normal renal tissue and various RCC histological types in cohort A, as shown in figure 1 . Panels A and B demonstrate a positive PAX-8

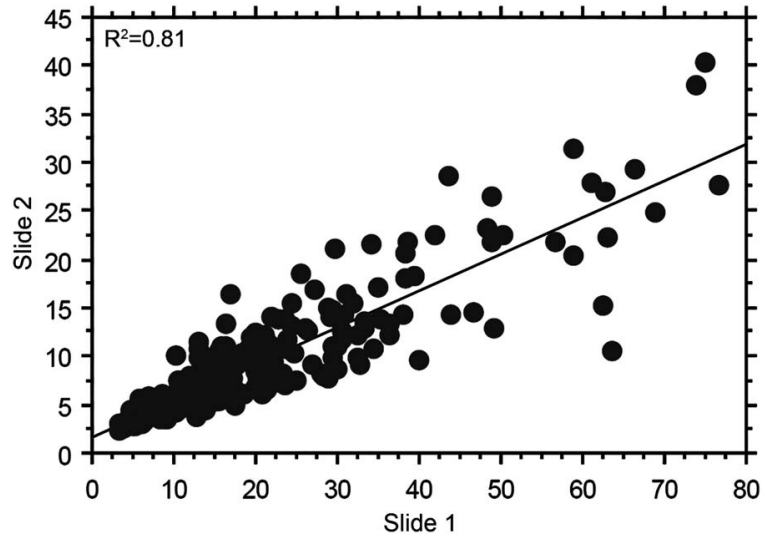

Figure 2 Pearson's correlation between slide 1 and slide 2 of cohort A reveals strong correlation in scores $(R=0.9)$. histo spot with an AQUA score of 56.05, while Panels C and D depict a negative PAX-8 histo spot with an AQUA score of 5.77. PAX-8 antibody demonstrated strong correlation in individual tumours on different TMA slides, as shown by the Pearson's correlation coefficient $(\mathrm{R}=0.9$, figure 2$)$.

The data distribution for PAX-8 AQUA scores was normal. In tumour tissue, AQUA scores ranged from 3.219 to 76.684 with a mean score of 17.651 (median score: 12.982) (table 1). A similar distribution was seen in normal renal specimens where AQUA scores ranged from 2.42 to 74.300 with a mean score of 20.455 (median score: 17.834). The distribution of AQUA scores in each histological subtype is provided in table 1. Paired $t$ test analysis revealed that clear cell RCC had significantly less PAX-8 expression than normal tissue $(p<0.0001$, figure $3 \mathrm{~A})$. There were no statistically significant differences in expression observed between normal tissue and chromophobe RCC $(p=0.1715)$ and normal tissue and oncocytomas $(p=0.2574$, table 2). There was a trend for higher PAX-8 expression in papillary RCC compared with normal tissue, but this failed to reach significance $(p=0.0748$, figure $3 B)$. Fisher's protected least significant difference testing demonstrated that papillary tumours

Table 1 PAX-8 staining in primary RCC and normal tissue (Cohort A)

\begin{tabular}{lrccccc}
\hline Tissue type & $\begin{array}{l}\text { Positive } \\
\text { cases (\%) }\end{array}$ & $\begin{array}{l}\text { Negative } \\
\text { cases }\end{array}$ & $\begin{array}{l}\text { Total } \\
\text { cases }\end{array}$ & $\begin{array}{l}\text { AQUA } \\
\text { mean }\end{array}$ & $\begin{array}{l}\text { AQUA } \\
\text { STD }\end{array}$ & $\begin{array}{l}\text { AQUA } \\
\text { median }\end{array}$ \\
\hline Chromophobe & $6(100 \%)$ & 0 & 6 & 12.328 & 4.616 & 12.195 \\
RCC & & & & & & \\
Clear cell RCC & $165(80 \%)$ & 42 & 207 & 15.234 & 12.552 & 10.492 \\
Oncocytoma & $17(94 \%)$ & 1 & 18 & 26.319 & 14.968 & 21.111 \\
Papillary RCC & $39(95 \%)$ & 2 & 41 & 26.827 & 19.736 & 20.252 \\
Total tumour & $227(83 \%)$ & 45 & 272 & 17.651 & 14.647 & 12.982 \\
Normal tissue & $146(96 \%)$ & 6 & 152 & 20.455 & 12.205 & 17.834 \\
\hline
\end{tabular}

AQUA, automated quantitative analysis; RCC, renal cell carcinoma. 

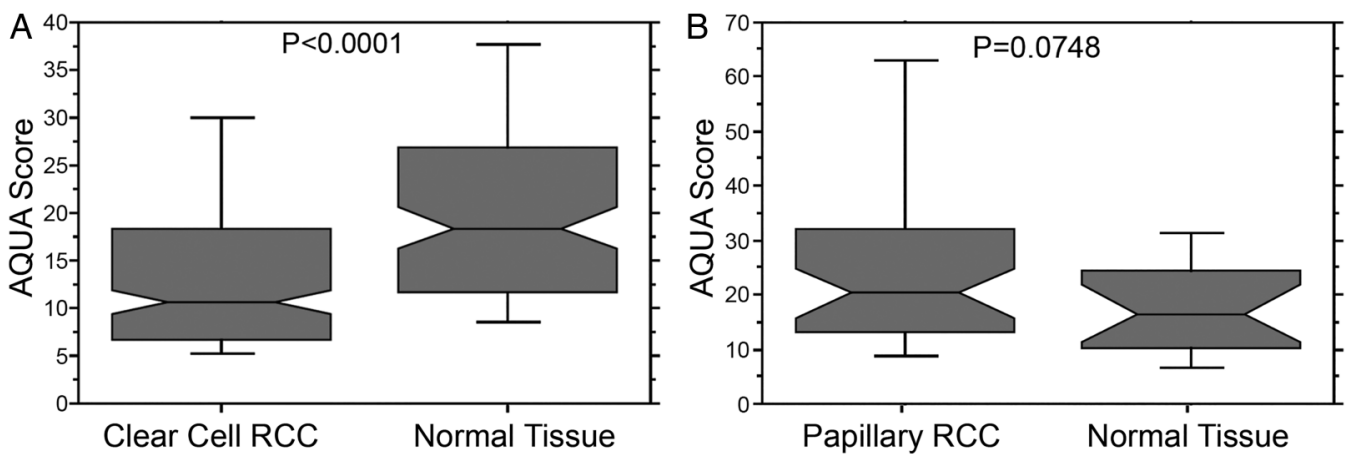

Figure 3 (A) Comparison of the median PAX-8 expression levels in clear cell renal cell carcinoma (RCC) versus normal tissue as visualised by box plots. Normal tissue has significantly higher PAX-8 expression than clear cell RCC tissue $(p<0.0001)$. (B) Comparison of PAX-8 expression in papillary RCC versus normal tissue as visualised by box plot. Papillary RCC trends towards having more expression of PAX-8 than normal tissue $(p=0.0748)$. For the box plot the box represents the range from the 25 th to the 75 th centiles. The centre line represents the median and the notches showing the $95 \% \mathrm{Cl}$ for the median. The whiskers demonstrate the 90th and the 10th centiles. AQUA, automated quantitative analysis.

and oncocytomas had higher PAX-8 expression than clear cell and chromophobe tumours (table 3). PAX-8 AQUA scores for clear cell and papillary RCCs were evaluated in relation to clinicopathological variables. No association was observed with regard to tumour stage, nuclear grade, sex and age. Analysis of the PAX-8 expression, as a continuous variable, in the Cox univariate analysis, failed to detect an association with 10-year disease-specific survival for either clear cell $(\mathrm{n}=189$, deaths $=52$, relative risk $=1.001$ (95\% CI 0.975 to 1.027$), \mathrm{p}=0.9692)$ or papillary RCC $(\mathrm{n}=39$, deaths $=9$, relative risk $=0.949(95 \%$ CI 0.890 to 1.012$), \mathrm{p}=0.1102$ ).

Cohort B was used to evaluate PAX-8 expression in matched primary tumour and metastatic sites. AQUA scores for the primary tumour and metastatic sites ranged from 4.939 to 30.998 with a mean of 11.599 (median score: 9.764) and from 4.834 to 56.961 with a mean of 17.997 (median score: 14.182), respectively (table 4). By ANOVA we found that PAX-8 expression is significantly higher in metastatic than in primary sites $(p=0.0047$, figure $4 A)$. Overall, 29 of 38 primary tumours were positive for PAX-8, while 9 were negative (table 4). Thirty-one of 33 metastatic tumours were positive for PAX-8, while 2 were negative (table 4). Of the assessable cases with corresponding matched tissue, 22/24 PAX-8 positive primary tumours had positive expression in the metastatic site. There was no statistical difference in positivity between the matched pairs $\left(\chi^{2} p=0.274\right.$, figure $\left.4 B\right)$.

\section{DISCUSSION}

Tissue evaluation by light microscopy of tissue core does not always guarantee the successful identification of the tumour type. Frequently, reliance on cellular morphology alone may be

Table 2 Paired $t$ test for PAX-8 expression between RCC subtypes and normal tissue

\begin{tabular}{lrrr}
\hline & Mean difference & t statistic & p Value \\
\hline Chromophobe RCC, normal tissue & -11.664 & -2.093 & 0.1715 \\
Clear cell RCC, normal tissue & -5.538 & -4.277 & $<0.0001$ \\
Oncocytomas, normal tissue & 9.792 & 1.233 & 0.2574 \\
Papillary RCC, normal tissue & 9.971 & 1.906 & 0.0748 \\
Tumour tissue, normal tissue & -2.753 & -1.983 & $\mathbf{0 . 0 4 9 4}$ \\
\hline Associations with $p<0.05$ are in bold. & & & \\
RCC, renal cell carcinoma. & & &
\end{tabular}

insufficient to help distinguish renal origin from other sites of disease or determine the renal histological subtype. Often the sample may be too small or too poorly differentiated to make a definitive diagnosis by morphology. A recent survey performed by the International Society of Urologic Pathologists found that a majority (nearly 90\%) of pathologists report using various immunostains to confirm the diagnosis of mRCC. ${ }^{3}$ There was consensus among International Society of Urologic Pathologists respondents that immunohistochemistry should be performed prior to confirming a diagnosis of an unclassified renal tumour. While many reported using a variety of well-characterised markers such as CK7, vimentin, CD10 and RCC marker, the majority of respondents reported that the most useful immunostains were PAX-2 and PAX- $8 .{ }^{3}$

Most genitourinary malignancies rely on diagnostic material to dictate the course of treatment. Kidney cancer is unique in that there is no consensus on the role of biopsy of the primary and distant metastases. Currently the American Urologic Association guidelines suggest renal mass biopsy when there are clinical or radiological concerns for lymphoma or metastasis to the kidney from a different solid tumour. ${ }^{30}$ In the setting of a possible nonrenal malignancy, a biopsy in conjunction with molecular markers could alter management, as a renal mass may represent a metastatic site of disease nearly $20 \%$ of the time. ${ }^{31}$ In other situations, such as prior to cytoreductive nephrectomy, some experts have suggested that tissue assessment of either the primary or a distant site may be useful to help defer surgery. ${ }^{32}$ If morphological or IHC characteristics can confirm the presence of a nonrenal malignancy, this likely will alter management as there is limited role of debulking for most non-RCC tumours.

Table 3 Fisher's PLSD test comparing PAX-8 expression in RCC subtypes

\begin{tabular}{lcr}
\hline & Mean difference & p Value \\
\hline Chromophobe RCC, clear cell RCC & -2.906 & 0.6146 \\
Chromophobe RCC, oncocytoma & -13.991 & 0.0339 \\
Chromophobe RCC, papillary RCC & -14.499 & 0.0179 \\
Clear cell RCC, oncocytoma & -11.085 & $\mathbf{0 . 0 0 1 3}$ \\
Clear cell RCC, papillary RCC & -11.592 & $<0.0001$ \\
Oncocytomas, papillary RCC & -0.508 & 0.8975 \\
\hline Associations with p<0.05 are bolded. &
\end{tabular}


Table 4 PAX-8 staining in primary and metastatic renal cell carcinoma (RCC) (Cohort B)

\begin{tabular}{llllllr}
\hline Tissue type & $\begin{array}{l}\text { Positive } \\
\text { cases } \\
(\%)\end{array}$ & $\begin{array}{l}\text { Negative } \\
\text { cases }\end{array}$ & $\begin{array}{l}\text { Total } \\
\text { cases }\end{array}$ & $\begin{array}{l}\text { AQUA } \\
\text { mean }\end{array}$ & \multicolumn{1}{l}{$\begin{array}{l}\text { AQUA } \\
\text { STD }\end{array}$} & $\begin{array}{l}\text { AQUA } \\
\text { median }\end{array}$ \\
\hline Primary RCC & $29(76 \%)$ & 9 & 38 & 11.599 & 6.507 & 9.764 \\
Metastatic RCC & $31(97 \%)$ & 2 & 33 & 17.997 & 11.572 & 14.182 \\
Total RCC & $60(85 \%)$ & 11 & 71 & 14.573 & 9.692 & 12.982 \\
\hline
\end{tabular}

AQUA, automated quantitative analysis.

As the role of molecular markers used for tissue confirmation on nephrectomy specimens and biopsy continues to expand, it is important to understand the reliability of markers used in clinical practice. In this study, two specially designed TMAs were analysed to evaluate the PAX-8 expression with quantitative immunohistochemical assessment. Our study has several
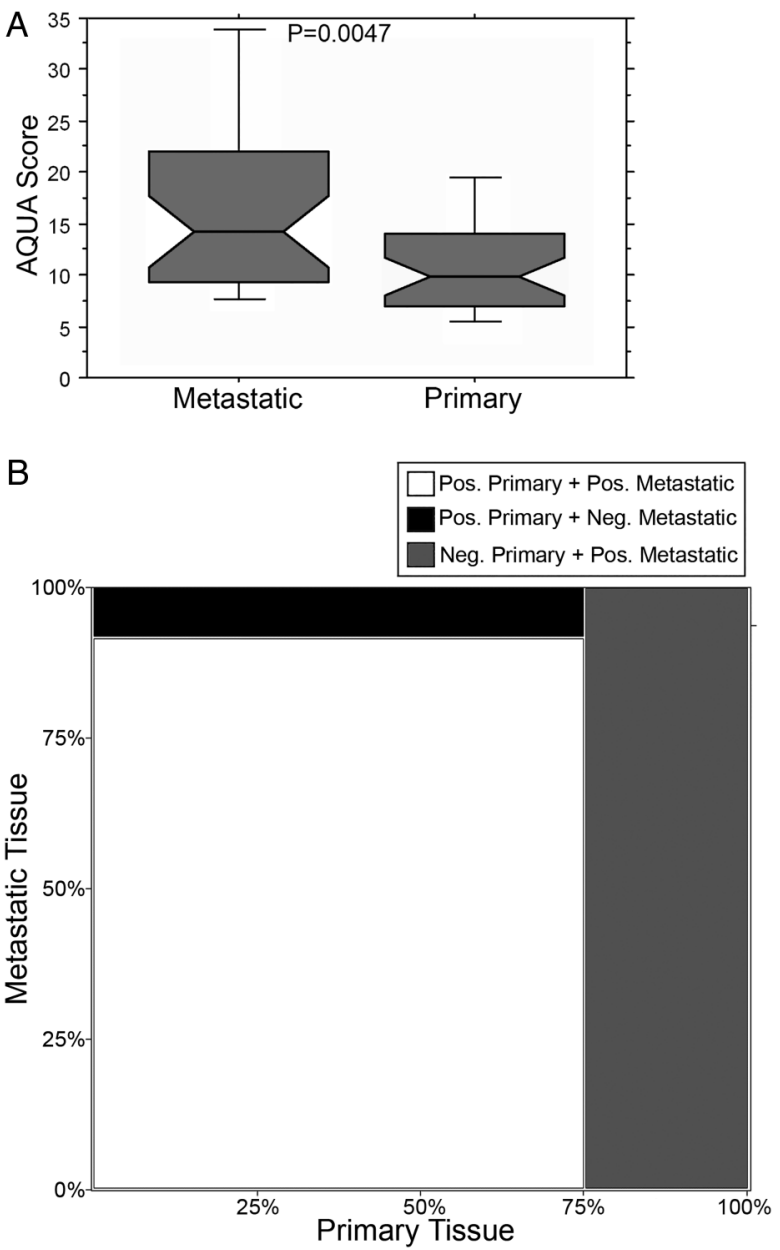

Figure 4 (A) Differences between the median PAX-8 expression levels in primary and metastatic sites by means plots. PAX- 8 expression is significantly higher in metastatic than primary sites. For the box plot the box represents the range from the 25th to the 75th centiles. The centre line represents the median and the notches showing the $95 \% \mathrm{Cl}$ for the median. The whiskers demonstrate the 90th and the 10th centiles. (B) $\chi^{2}$ analysis of PAX-8 expression in primary and matched metastatic samples. Of the assessable cases with corresponding matched tissue, 22/24 PAX-8 positive primary tumours had positive expression in the metastatic site. There was no statistical difference in positivity between the matched pairs $\left(\chi^{2} p=0.274\right)$. important findings regarding this diagnostic marker for RCC. First, in normal kidney tissue, we confirm that PAX-8 is highly expressed and positive in $96 \%$ of samples. Second, we found that renal tumours overall were less frequently positive for PAX-8 expression (83\% of cases) and had a significantly lower intensity of staining when compared with normal tissue. The primary renal tumour positivity is less than the $>90 \%$ reported in several series. ${ }^{18} 22$ Third, we demonstrate that among renal tumours, PAX-8 expression varies significantly by histological subtype and compared with normal kidney, may have either similar or lower PAX-8 expression.

The most common histological subtype, clear cell RCC, had significantly lower expression than normal kidney and papillary RCCs. Our assessment of clear cell RCC PAX-8 positivity, $80 \%$ $(165 / 207)$, differs from prior reports that mention positivity to be between $91 \%$ and $98 \% .^{13}{ }^{22}$ For papillary RCC, PAX-8 expression was positive in 95\% (39/41) of cases which is consistent with the $71-100 \%$ positivity in the literature. ${ }^{13} 2022$ Prior findings raised questions regarding the utility of PAX-8 expression in chromophobe RCC as positivity was reported to be as low as $57 \%$ with strong expression in only $9 \%$ of cases. $^{13} 2022$ We only had six chromophobe RCCs in our cohort, yet all had PAX-8 expression, and the level of expression, while lower than papillary RCC and oncocytoma, was similar to normal kidney and clear cell RCCs.

A recent report found decreased PAX-8 expression with higher grade renal tumours. ${ }^{20}$ Such a finding could perhaps have important implications on the utility of this marker to detect more aggressive malignancies. However, our quantitative evaluation of PAX-8 expression with clinicopathological variables found no significant association with stage or grade, similar to prior reports showing no correlation of grade to marker positivity. ${ }^{18}$ Additionally, other reports demonstrated that PAX-8 expression is positive in nearly 90\% of metastatic sites of disease. ${ }^{20}$ In the matched metastatic and primary RCC array (Cohort B), more intense PAX-8 immunostaining was actually seen in the metastatic specimens compared with the matched primary tumour. Our data aligns with recent work in a smaller cohort $(n=15)$ that demonstrated higher levels of PAX-8 in metastatic tissue than in primary tissue. ${ }^{33}$ However, while quantitatively higher, there was no difference in positivity between the matched pairs, suggesting that in cases where only primary or only metastatic tissue is available, either specimen type can be used to make a correct kidney cancer diagnosis.

PAX-8 expression has been evaluated in other large patient cohorts, however this is the first study to use an objective, automated method for assessment of expression. Subjectivity of pathologist-based scoring might explain the difference in the per cent of positive cases in the clear cell population. In addition, in other cohorts non-automated methods have analysed PAX-8 expression, but all were conducted using tissue sections rather than a TMA. ${ }^{16} 1920$ Our separate and unique TMAs allowed us to compare various tissue types including a large cohort of primary renal tumours and normal kidney tissue as well as matched primary and metastatic tissues. The strong correlation between matching spots from the same tumour suggests that a core is an adequate indicator of expression in an entire specimen. While TMA design is not without limitations due to tissue heterogeneity, multiple cores from different tumour regions improves the assessment of protein expression. ${ }^{34}$

\section{CONCLUSIONS}

The role of molecular markers is evolving in the diagnosis and management of kidney cancer. PAX- 8 is one of the most 
commonly used markers to aid in the diagnosis of RCC. We confirm that PAX-8 expression is a useful marker for the primary tumour and distant sites. PAX-8 expression is more commonly positive in normal parenchyma than in renal tumours and there are differences in expression between histological subtypes. However, compared with normal tissue and other histological types, clear cell renal tumours have lower PAX-8 expression and are less often positive (80\% of cases), therefore, lack of expression does not definitively preclude a renal origin. We did not observe an association of PAX-8 expression with clinocopathological variables.

\section{Take home messages}

- Pathologists frequently rely on various immunohistochemical markers to confirm the diagnosis of metastatic renal cell carcinoma (RCC) and or an unclassified renal tumour.

- PAX-8 is a marker of renal organogenesis and is one of the most commonly used markers for RCC.

- Using automated quantitative analysis, we demonstrate that PAX-8 is a useful marker for primary tumours and distant sites of disease.

- Clear cell RCC has lower PAX-8 expression and is less often positive and therefore, the lack of expression alone should not rule out a tumour of renal origin.

- PAX-8 expression is more commonly positive in normal parenchyma than in renal tumours.

- Histological subtypes have different levels of PAX-8 expression.

- There is no association of PAX-8 expression with clinocopathological variables.

Contributors Conception and design: HMK, LBJ and BS. Provision of study materials or patients: RLC and AJA. Collection and assembly of data: LBJ and MLB. Data analysis/interpretation: BS, LBJ, HMK, AJA, MLB and RLC. Manuscript writing: $M L B, B S, L B J$ and HMK. Final approval of manuscript: BS, LBJ, HMK, AJA, MLB and RLC.

Funding This work was supported by NIH grants R0-1 CA158167 (to HMK), K24CA172123 (to HMK), and by American Cancer Society Award M130572 (to HMK). This publication was also made possible by CTSA Grant Number KL2 TR000140 (LBJ) from the National Center for Research Resources (NCRR) and the National Center for Advancing Translational Science (NCATS), components of the National Institutes of Health (NIH), and NIH roadmap for Medical Research. US Department of Health and Human Services-National Institutes of Health-NIH Office of the Director (K24CA172123, KL2 TR000140, R0-1 CA158167)

Competing interests None.

Provenance and peer review Not commissioned; externally peer reviewed.

Data sharing statement Raw data for AQUA expression is available upon request.

\section{REFERENCES}

1 (NCCN) NCCN. Kidney Cancer. NCCN Clinical Practice Guidelines in Oncology, 2012

2 Shuch B, Said J, LaRochelle JC, et al. Histologic evaluation of metastases in rena cell carcinoma with sarcomatoid transformation and its implications for systemic therapy. Cancer 2010;116:616-24.

3 Tan $\mathrm{PH}$, Cheng L, Rioux-Leclercq N, et al. Renal tumors: diagnostic and prognostic biomarkers. Am J Surg Pathol 2013;37:1518-31.

4 Skinnider BF, Amin MB. An immunohistochemical approach to the differential diagnosis of renal tumors. Semin Diagn Pathol 2005:22:51-68.

5 Sangoi AR, Karamchandani J, Kim J, et al. The use of immunohistochemistry in the diagnosis of metastatic clear cell renal cell carcinoma: a review of PAX-8, PAX-2, hKIM-1, RCCma, and CD10. Adv Anat Pathol 2010;17:377-93.
6 Shen SS, Truong LD, Scarpelli M, et al. Role of immunohistochemistry in diagnosing renal neoplasms: when is it really useful? Arch Pathol Lab Med 2012:136:410-17.

7 Ordonez NG. Value of PAX 8 immunostaining in tumor diagnosis: a review and update. Adv Anat Pathol 2012;19:140-51.

8 Shuch B, Li Z, Belldegrun AS. Carbonic anhydrase IX and renal cell carcinoma: prognosis, response to systemic therapy, and future vaccine strategies. BJU Int 2008;101(Suppl 4):25-30.

9 Bouchard M, Souabni A, Mandler M, et al. Nephric lineage specification by Pax2 and Pax8. Genes Dev 2002;16:2958-70.

10 Lang D, Powell SK, Plummer RS, et al. PAX genes: roles in development, pathophysiology, and cancer. Biochem Pharmacol 2007;73:1-14.

11 Narlis M, Grote D, Gaitan Y, et al. Pax2 and pax8 regulate branching morphogenesis and nephron differentiation in the developing kidney. J Am SoC Nephrol 2007;18:1121-9.

12 Poleev $\mathrm{A}$, Fickenscher $\mathrm{H}$, Mundlos $\mathrm{S}$, et al. PAX8, a human paired box gene: isolation and expression in developing thyroid, kidney and Wilms' tumors. Development 1992:116:611-23.

13 Tong GX, Yu WM, Beaubier NT, et al. Expression of PAX8 in normal and neoplastic renal tissues: an immunohistochemical study. Mod Pathol 2009:22:1218-27.

14 Albadine R, Schultz L, Illei P, et al. PAX8 (+)/p63 (-) immunostaining pattern in renal collecting duct carcinoma $(C D C)$ : a useful immunoprofile in the differential diagnosis of CDC versus urothelial carcinoma of upper urinary tract. Am J Surg Pathol 2010;34:965-9.

15 Carney EM, Banerjee $\mathrm{P}$, Ellis $\mathrm{CL}$, et al. PAX2(-)/PAX8(-)/inhibin $\mathrm{A}(+)$ immunoprofile in hemangioblastoma: a helpful combination in the differential diagnosis with metastatic clear cell renal cell carcinoma to the central nervous system. Am J Sur Pathol 2011;35:262-7.

16 Hu Y, Hartmann A, Stoehr C, et al. PAX8 is expressed in the majority of renal epithelial neoplasms: an immunohistochemical study of 223 cases using a mouse monoclonal antibody. J Clin Pathol 2012;65:254-6.

17 Knoepp SM, Kunju LP, Roh MH. Utility of PAX8 and PAX2 immunohistochemistry in the identification of renal cell carcinoma in diagnostic cytology. Diagn Cytopathol 2012:40:667-72

18 Laury AR, Perets $\mathrm{R}$, Piao $\mathrm{H}$, et al. A comprehensive analysis of PAX8 expression in human epithelial tumors. Am J Surg Pathol 2011;35:816-26.

19 Ozcan A, de la Roza G, Ro JY, et al. PAX2 and PAX8 expression in primary and metastatic renal tumors: a comprehensive comparison. Arch Pathol Lab Med 2012;136:1541-51.

20 Ozcan A, Shen SS, Hamilton C, et al. PAX 8 expression in non-neoplastic tissues, primary tumors, and metastatic tumors: a comprehensive immunohistochemical study. Mod Pathol 2011;24:751-64.

21 Sangoi AR, Fujiwara M, West RB, et al. Immunohistochemical distinction of primary adrenal cortical lesions from metastatic clear cell renal cell carcinoma: a study of 248 cases. Am J Surg Pathol 2011;35:678-86.

22 Tacha D, Zhou D, Cheng L. Expression of PAX8 in normal and neoplastic tissues: a comprehensive immunohistochemical study. Appl Immunohistochem Mol Morphol 2011;19:293-9

23 Carvalho JC, Thomas DG, McHugh JB, et al. p63, CK7, PAX8 and INI-1: an optimal immunohistochemical panel to distinguish poorly differentiated urothelial cell carcinoma from high-grade tumours of the renal collecting system. Histopathology 2012:60:597-608

24 Gibney GT, Aziz SA, Camp RL, et al. c-Met is a prognostic marker and potential therapeutic target in clear cell renal cell carcinoma. Ann Oncol 2013;24:343-9.

25 Aziz SA, Sznol J, Adeniran A, et al. Vascularity of primary and metastatic renal cell carcinoma specimens. J Trans/ Med 2013;11:15.

26 Kluger HM, Siddiqui SF, Angeletti C, et al. Classification of renal cell carcinoma based on expression of VEGF and VEGF receptors in both tumor cells and endothelial cells. Lab Invest 2008;88:962-72.

27 Camp RL, Chung GG, Rimm DL. Automated subcellular localization and quantification of protein expression in tissue microarrays. Nat Med 2002;8:1323-7.

28 Jilaveanu LB, Shuch B, Zito CR, et al. PD-L1 expression in clear cell renal cell carcinoma: an analysis of nephrectomy and sites of metastases. J Cancer 2014;5:166-72.

29 Scouten WT, Patel A, Terrell R, et al. Cytoplasmic localization of the paired box gene, Pax-8, is found in pediatric thyroid cancer and may be associated with a greater risk of recurrence. Thyroid 2004;14:1037-46.

30 Campbell SC, Novick AC, Belldegrun A, et al. Guideline for management of the clinical T1 renal mass. J Urol 2009;182:1271-9.

31 Sanchez-Ortiz RF, Madsen LT, Bermejo CE, et al. A renal mass in the setting of a nonrenal malignancy: when is a renal tumor biopsy appropriate? Cancer 2004:101:2195-201.

32 Abel E, Culp S, Margulis V, et al. Use of percutaneous primary tumor biopsy to predict high-risk pathologic features in patients with metastatic renal cell carcinoma. ASCO: GU Cancer Symposium 2009. 2009; Abstract No: 311

33 Pan Z, Grizzle W, Hameed O. Significant variation of immunohistochemical marke expression in paired primary and metastatic clear cell renal cell carcinomas. Am J Clin Pathol 2013;140:410-18.

34 Eckel-Passow, JE, Lohse CM, Sheinin $Y$, et al. Tissue microarrays: one size does not fit all. Diagnostic Pathology 2010;5:48 